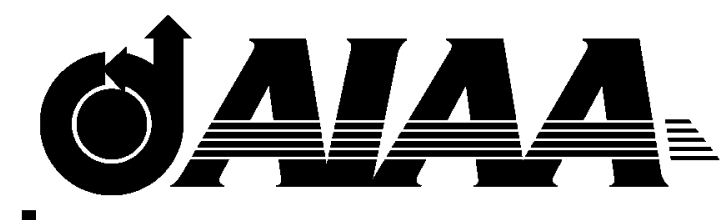

\title{
AIAA 2002-3286
}

Adjoint-Based, Three-Dimensional Error Prediction and Grid Adaptation

Michael A. Park

NASA Langley Research Center, Hampton, VA 23681

\section{2nd Fluid Dynamics Conference 24-27 June 2002 St. Louis, MO}




\title{
Adjoint-Based, Three-Dimensional Error Prediction and Grid Adaptation
}

\author{
Michael A. Park * \\ NASA Langley Research Center, Hampton, VA 23681
}

\begin{abstract}
Engineering computational fluid dynamics (CFD) analysis and design applications focus on output functions (e.g., lift, drag). Errors in these output functions are generally unknown and conservatively accurate solutions may be computed. Computable error estimates can offer the possibility to minimize computational work for a prescribed error tolerance. Such an estimate can be computed by solving the flow equations and the linear adjoint problem for the functional of interest. The computational mesh can be modified to minimize the uncertainty of a computed error estimate. This robust mesh-adaptation procedure automatically terminates when the simulation is within a user specified error tolerance. This procedure for estimating and adapting to error in a functional is demonstrated for three-dimensional Euler problems. An adaptive mesh procedure that links to a Computer Aided Design (CAD) surface representation is demonstrated for wing, wing-body, and extruded high lift airfoil configurations. The error estimation and adaptation procedure yielded corrected functions that are as accurate as functions calculated on uniformly refined grids with ten times as many grid points.
\end{abstract}

\section{Introduction}

Engineering problems commonly require computational fluid dynamics (CFD) solutions with functional outputs of specified accuracy. The computational resources available for these solutions are often limited and errors in solutions and outputs are often unknown. CFD solutions may be computed with an unnecessarily large number of grid points (and associated high cost) to ensure that the outputs are computed to within a required accuracy. A method to estimate the error present in a computed functional offers the possibility to avoid the use of overly refined grids to guarantee accuracy.

Unstructured grid technology promises easier initial grid generation for novel complex three-dimensional

\footnotetext{
* Research Scientist, Computational Modeling and Simulation Branch, m.a.park@larc.nasa.gov

Copyright (C) 2002 by the American Institute of Aeronautics and Astronautics, Inc. No copyright is asserted in the United States under Title 17, U.S. Code. The U.S. Government has a royaltyfree license to exercise all rights under the copyright claimed herein for Governmental Purposes. All other rights are reserved by the copyright owner.
}

(3D) configurations compared with structured grid techniques. The use of unstructured grid technology for CFD simulations allows more freedom in adapting the discretization of the meshes to improve the fidelity of the simulation. Many previous efforts have attempted to tailor the discretizations of unstructured meshes to increase solution accuracy while reducing computational cost..$^{1-8}$

Most of these adaptive methods focus on modifying discretizations to reduce local equation errors. These local errors are not guaranteed to directly impact error in global output functions. These methods, often referred to as feature-based adaptation, focus on resolving discontinuities or strong gradients in the flow field. Flow features (e.g., shocks) can be in the incorrect location due to errors elsewhere in the flow field. Also, resolving the flow in a location may have a minimal effect upon the output function (e.g., a downstream shock).

If the flow equations are linearized about the flow solution, the solution of a linear dual problem can yield a direct measure of the impact of local primal (flow equation) residual on a selected functional output. The combination of the primal and dual problems can also be applied to yield a correction to a specified functional on a given mesh. If a specified error tolerance in an output function is required, the cost of computing a CFD solution can be minimized by adapting the discretization of the problem to directly minimize uncertainties in the corrected output function of interest. Also, the entire adaptive simulation can be terminated when the predicted error is equal to a specified tolerance, preventing the waste of computational work on an overly large mesh.

There are many examples of these techniques in the finite element communities. ${ }^{9,10}$ Pierce and Giles ${ }^{11}$ applied these methods to finite-volume discretizations. Venditti and Darmofal ${ }^{12,13}$ have demonstrated these methods for compressible two-dimensional (2D) inviscid and viscous flow solutions. The present study is essentially a $3 \mathrm{D}$ extension of the methods of Venditti and Darmofal ${ }^{13}$ that adapts the mesh to reduce uncertainty in an error correction. Müller and Giles ${ }^{14}$ have also presented results for a similar approach utilizing a different adaptation parameter that directly targets the error correction.

The use of adjoint variables (solution of the dual 
problem) is an efficient method for computing derivatives of a functional of interest for gradient-based design methods. Some examples of discrete adjoint design methods are given in Nielsen and Anderson. ${ }^{15,16}$ The discrete dual problem for adjoint variables can be expensive. However, the adjoint solution is already available for use during the aerodynamic design process, so it could be employed for simultaneous design, error prediction, and grid adaptation.

The combination of adjoint-based grid adaptation and design techniques can yield an attractive tool for the aerodynamic design of new configurations. Adjoint-based error prediction and adaptation can yield smaller meshes than traditional feature-based schemes with computable error estimates on output functions. Design processes require analysis and derivative evaluation tools that operate with minimal human interaction. Robust, automatic adaptation techniques enable the increased use of nonlinear flow calculations in larger multidisciplinary design frameworks. These new techniques will enable efficient analysis for existing configurations and expanded exploration of design spaces for new configurations.

\section{Flow Equations}

The FUN3D ${ }^{17-19 \dagger}$ (Fully Unstructured NavierStokes Three-Dimensional) suite of codes is employed in this study. The compressible flow solver employs an unstructured finite-volume tetrahedral method for conserved variables, $\mathbf{Q}$, i.e.,

$$
\mathbf{Q}=[\rho \rho u \rho \rho w E]^{T}
$$

where $\rho$ is density, $u, v$, and $w$ are velocity, and $E$ is total energy per unit volume. The incompressible flow solver employs the following state variables:

$$
\mathbf{Q}=\left[\begin{array}{llll}
p & u & v & w
\end{array}\right]^{T}
$$

where $p$ is pressure. The node-based variables $\mathbf{Q}$ are computed by driving the flow equation residual $\mathbf{R}$ to steady-state with an implicit point-iterative method. The code is able to solve incompressible, Euler, and Reynolds-Averaged Navier-Stokes (RANS) flow equations loosely coupled to the Spalart-Allmaras ${ }^{20}$ oneequation turbulence model. The present study employs only the Euler and incompressible equations. The solution of $\mathbf{Q}$ allows the calculation of integral quantities $f$ (e.g., lift, drag). To speed execution, the problem domain is decomposed and the flow and the adjoint problems are solved with a parallel execution scheme utilizing the Message Passing Interface (MPI) standard. The FUN3D suite of codes is being extended to the HEFSS (High Energy Flow Solver Synthesis) ${ }^{21}$ modular framework of FORTRAN 90 shared libraries.

\footnotetext{
$t_{\text {http: / / fun3d. larc.nasa.gov }}$
}

\section{Adjoint Equations}

After the flow solution is known, the discrete adjoint equations ${ }^{15,18,19}$ are solved to complete the dual problem. The first step is to linearize the flow equation residual $\mathbf{R}$ and output function $f$ with respect to the flow solution $\mathbf{Q}$. After this linearization, an adjoint variable $\lambda$ is solved for each of the flow equations.

An abbreviated derivation, adapted from Taylor et al., ${ }^{22}$ is below. The chain rule for the linearized output function is

$$
\frac{\partial f}{\partial \mathbf{Q}}=\left(\frac{\partial f}{\partial \mathbf{R}}\right)^{T} \frac{\partial \mathbf{R}}{\partial \mathbf{Q}}
$$

The adjoint variable $\lambda$ is defined as the effect of the flow residual on the output function:

$$
\frac{\partial f}{\partial \mathbf{R}}=\lambda
$$

A set of linear equations is solved to find $\lambda$ :

$$
\left(\frac{\partial \mathbf{R}}{\partial \mathbf{Q}}\right)^{T} \lambda=\left(\frac{\partial f}{\partial \mathbf{Q}}\right)^{T}
$$

After the flow solution is known, this set of linear equations is solved with GMRES. ${ }^{23}$ See Refs. 15,16 , and 19 for details. A implicit point-iterative time-marching method is employed to compute the adjoint solution for the high lift configuration. ${ }^{24-26}$

\section{Error Correction}

The error prediction and correction scheme is taken from Ref. 13. With a solution on a mesh of reasonable size $\mathbf{Q}^{0}$, it is desirable to predict the value of an output function evaluated with a solution on a much finer mesh $f\left(\mathbf{Q}^{*}\right)$. This prediction can be computed without the solution on this finer mesh when the adjoint solution on this reasonable mesh $\lambda^{0}$ is utilized. The full derivation of the error correction term is available in Refs. 11, 13,14. An abbreviated derivation is presented by expanding the Taylor series about $f\left(\mathbf{Q}^{0}\right)$, i.e.,

$$
f\left(\mathbf{Q}^{*}\right)=f\left(\mathbf{Q}^{0}\right)+\left(\left.\frac{\partial f}{\partial \mathbf{R}}\right|_{0}\right)^{T}\left(\mathbf{R}\left(\mathbf{Q}^{*}\right)-\mathbf{R}\left(\mathbf{Q}^{0}\right)\right)+\ldots
$$

Employing eq. (4) and assuming that the residual on the much finer mesh is zero yields an improved estimate for the functional of interest $f_{\text {est }}$ :

$$
\begin{gathered}
\left.\frac{\partial f}{\partial \mathbf{R}}\right|_{0}=\lambda^{0} \\
\mathbf{R}\left(\mathbf{Q}^{*}\right)=0 \\
f\left(\mathbf{Q}^{*}\right) \approx f_{\text {est }}=f\left(\mathbf{Q}^{0}\right)-\left(\lambda^{0}\right)^{T} \mathbf{R}\left(\mathbf{Q}^{0}\right)
\end{gathered}
$$

To improve the prediction of the functional $f_{\text {est }}, \mathbf{Q}^{0}$ and $\lambda^{0}$ can be interpolated to an embedded mesh. Interpolation is performed in two ways for this study: 
a linear interpolation $\left(\mathbf{Q}^{L}, \lambda^{L}\right)$ and a higher order interpolation $\left(\mathbf{Q}^{H}, \lambda^{H}\right)$. Details of this interpolation and the construction of this embedded mesh are in the "Interpolation Techniques" and "Embedded Mesh" sections. Substituting these interpolated quantities into eq. (9) yields the linear and higher order functional estimates $f_{\text {est }}^{L}$ and $f_{\text {est }}^{H}$ :

$$
\begin{gathered}
f_{\text {est }}^{L}=f\left(\mathbf{Q}^{L}\right)-\left(\lambda^{L}\right)^{T} \mathbf{R}\left(\mathbf{Q}^{L}\right) \\
f_{\text {est }}^{H}=f\left(\mathbf{Q}^{H}\right)-\left(\lambda^{H}\right)^{T} \mathbf{R}\left(\mathbf{Q}^{H}\right)
\end{gathered}
$$

\section{Adaptation Parameter}

The adaptation parameter, also from Ref. 13, is intended to specify a grid spacing modification to reduce the uncertainty in the computed error prediction. The grid is not modified to directly reduce the computed error prediction (as in Ref. 14) because an estimate for the functional including this error term can be computed with eq. (9). Instead, targeting the uncertainty in this computed quantity is more effective and improves the robustness of the adaptive process. The error correction (eq. (9)) including the uncertainty in the dual solution is

$$
f\left(\mathbf{Q}^{0}\right)-f\left(\mathbf{Q}^{*}\right) \approx\left(\lambda^{0}\right)^{T} \mathbf{R}\left(\mathbf{Q}^{0}\right)+\left(\lambda^{*}-\lambda^{0}\right)^{T} \mathbf{R}\left(\mathbf{Q}^{0}\right)
$$

The uncertainty in the computed error correction is

$$
f_{e s t}-f\left(\mathbf{Q}^{*}\right) \approx\left(\lambda^{*}-\lambda^{0}\right)^{T} \mathbf{R}\left(\mathbf{Q}^{0}\right)
$$

The relation of the primal and dual problems ${ }^{11,13}$ yields another expression for the error correction uncertainty

$$
\left(\lambda^{*}-\lambda^{0}\right)^{T} \mathbf{R}\left(\mathbf{Q}^{0}\right)=\mathbf{R}_{\lambda}\left(\lambda^{0}\right)\left(\mathbf{Q}^{*}-\mathbf{Q}^{0}\right)^{T}
$$

Where $\mathbf{R}_{\lambda}(\lambda)$ is the residual of the dual problem:

$$
\mathbf{R}_{\lambda}(\lambda)=\left(\frac{\partial \mathbf{R}}{\partial \mathbf{Q}}\right)^{T}-\left(\frac{\partial f}{\partial \mathbf{Q}}\right)^{T} \lambda
$$

A computable term is found by using the interpolation error of $\lambda$ to replace $\left(\lambda^{*}-\lambda^{0}\right)$ and the interpolation error of $\mathbf{Q}$ to replace $\left(\mathbf{Q}^{*}-\mathbf{Q}^{0}\right)$. The higher order interpolate for $\mathbf{Q}^{0}$ and $\lambda^{0}$ is employed to improve prediction in place of the linear interpolate in Ref. 13. The interpolation error is expressed as the difference in the high-order and linear interpolated values:

$$
\begin{aligned}
\left(\lambda^{*}-\lambda^{0}\right) & \approx\left(\lambda^{H}-\lambda^{L}\right) \\
\left(\mathbf{Q}^{*}-\mathbf{Q}^{0}\right) & \approx\left(\mathbf{Q}^{H}-\mathbf{Q}^{L}\right)
\end{aligned}
$$

The average of the absolute values of the two uncertainty terms in eq. (14) yields the adaptation intensity I, which is computed for each equation on each embedded node:

$$
\mathbf{I}=\frac{\left|\left(\lambda^{H}-\lambda^{L}\right)^{T} \mathbf{R}\left(\mathbf{Q}^{H}\right)\right|+\left|\mathbf{R}_{\lambda}\left(\lambda^{H}\right)\left(\mathbf{Q}^{H}-\mathbf{Q}^{L}\right)^{T}\right|}{2}
$$

The intensity $\mathbf{I}$ is therefore the average of a primal residual weighted with a dual solution interpolation error and a dual problem residual weighted with a primal solution interpolation error. This form of the adaptation intensity tends to focus on the nonlinear contributions to the function error, which increases robustness of the adaptation method.

\section{Error Correction and Adaptation Process}

The error correction and adaptation process begins with an initial tetrahedral mesh, which can come from any mesh generation system. The state variables are computed as the nonlinear solution to the flow equations on the initial mesh. The adjoint variables are then computed with the linearized flow equations at the flow solution. These flow and adjoint solution procedures employ a parallel execution scheme. Then the global problem domain is reconstructed to facilitate the creation of a finer, embedded grid with interpolated primal and dual solutions.

\section{Embedded Mesh}

To compute the error prediction and the adaptation parameter a globally embedded or h-refined mesh is created. To construct the embedded mesh, a new node (open circle) is inserted at the midpoint of each existing edge that connects existing nodes (closed circles); see Fig. 1(a) and 1(b). Each existing tetrahedron is subdivided to reconnect these new nodes with eight interior tetrahedra. (Each of the existing boundary faces is also divided into four triangles.)

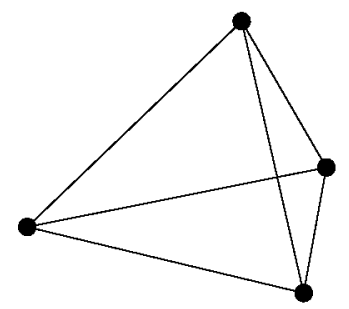

a) Original tetrahedron.

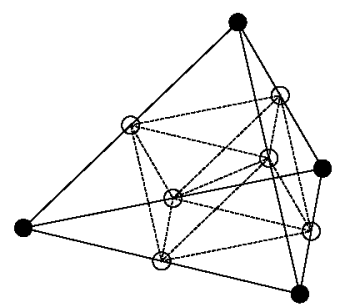

b) Embedded tetrahedron.
Fig. 1 Tetrahedron embedding process.

The four new tetrahedra constructed at the corners of the existing tetrahedron have the same shape as the original tetrahedron but are smaller in size. The construction of the four corner tetrahedra leaves an interior volume with eight faces, which is subdivided into four tetrahedra. The four interior tetrahedra have three unique configurations. The configuration with the lowest maximum dihedral angle is selected.

The new nodes are placed at the midpoints of edges during the mesh embedding process. The embedded nodes on the boundaries of the mesh may no longer remain on the original surface definition of the model. 
When the grid is adapted to improve the discretization, the surface fidelity of the mesh is maintained with boundary node projection.

\section{Interpolation Techniques}

The primal and dual solution variables $y$, which are all elements of $\mathbf{Q}$ and $\lambda$, are now interpolated to this embedded mesh. The value of the solution at each of the existing nodes is directly copied into the corresponding nodes of the embedded mesh. Each of the solution variables is interpolated in two ways to form the linear and the higher order reconstruction for the new nodes. The higher order reconstruction of the solution for the new nodes requires the computation of least-squares gradients ${ }^{17}$ at the existing nodes using the existing mesh and solution. To simplify the $3 \mathrm{D}$ interpolation, the interpolation is performed by independently examining each existing edge in the original mesh; thus the interpolation problem becomes onedimensional along each existing edge. Each existing edge has an existing node at each edge endpoint and a new node at the edge midpoint (see Fig. 2).

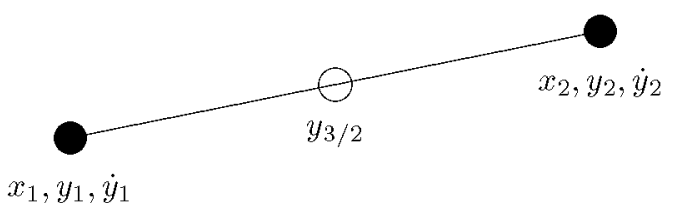

Fig. 2 New node and existing edge.

An edge has two $3 \mathrm{D}$ endpoints: $\mathbf{x}_{1}$ and $\mathbf{x}_{2}$. The vector that represents the length and direction of the edge is $\Delta \mathbf{x}=\mathbf{x}_{2}-\mathbf{x}_{1}$. The $3 \mathrm{D}$ least-squares gradient of the solution $\Delta y$ can be projected to a total derivative along the edge to facilitate interpolation by

$$
\dot{y}=\nabla y^{T} \Delta \mathbf{x}
$$

The new node interpolation $y_{3 / 2}$ can be expressed as a combination of the solution values and the derivatives at each endpoint $\left(y_{1}, \dot{y}_{1}, y_{2}\right.$, and $\left.\dot{y}_{2}\right)$. The linear interpolation $y_{3 / 2}^{L}$ is the average of the two end nodes

$$
y_{3 / 2}^{L}=\frac{y_{1}+y_{2}}{2}
$$

The higher order interpolation $y_{3 / 2}^{H}$ is found with a cubic fit of the endpoint data that is evaluated at the midpoint

$$
y_{3 / 2}^{H}=\frac{y_{1}+y_{2}}{2}+\frac{\dot{y}_{1}-\dot{y}_{2}}{8}
$$

Equation (21) is equivalent to a least-squares quadratic fit of the endpoint data that is evaluated at the edge midpoint.

At the completion of the grid embedding and interpolation step, the linear and higher order interpolated solutions to the primal and dual problems $\mathbf{Q}^{L}, \lambda^{L}$, $\mathbf{Q}^{H}$, and $\lambda^{H}$ are available to compute the error correction and adaptation parameter.

\section{Error Correction and Adaptation Parameter}

Once the new embedded grid is constructed with $\mathbf{Q}^{L}, \lambda^{L}, \mathbf{Q}^{H}$, and $\lambda^{H}$, it is partitioned to allow parallel calculation of the functional and flow and adjoint equation residuals. The flow and the adjoint equations are not iterated or solved on this embedded grid; the flow state and adjoint variables are interpolated from the original mesh. Therefore, the only computational costs on this larger embedded grid are function evaluations, flow and adjoint residual evaluations, and dot products of vectors. The linear and higher order error correction term, eq. (10) and (11), is computed at each node on the embedded mesh and summed over the entire mesh for all flow equations.

The adaptation intensity, eq. (18), is also computed at each embedded mesh node. At each node that is also present in the original mesh, the computed intensity is zero due to the chosen interpolation technique. The values of the lower and higher order interpolation schemes are the same at these existing nodes; thus $\left(\mathbf{Q}^{H}-\mathbf{Q}^{L}\right)$ and $\left(\lambda^{H}-\lambda^{L}\right)$ are exactly zero.

To specify the grid adaptation on the original mesh, the adaptation intensities must be reduced from the embedded mesh to the original mesh $\left(\mathbf{I}_{0}\right)$. The new nodes on the embedded mesh all lie on existing edges of the original mesh (see Fig. 1(b)). Therefore, to construct $\mathbf{I}_{0}$ the original mesh is examined one edge at a time (see Fig. 2). One half of the intensity computed at each new node (which is at the midpoint of these original edges) is added to each existing node at the endpoints of these edges. The intensities are also summed over the equations at this point, resulting in one intensity value for each original node.

The adaptation parameter, which has been reduced to the original mesh, is summed to find the global intensity $I_{g}=\sum \mathbf{I}_{0}$. The number of nodes in the original mesh $n$ and the user-specified error tolerance $t$ are combined to scale the adaptation intensity; that is

$$
\mathbf{I}_{s}=\frac{I_{g}}{t} \frac{n}{t} \mathbf{I}_{0}
$$

To perform the grid adaptation, the mesh is locally enriched in the location of nodes where the scaled intensity $\mathbf{I}_{s}$ is greater than a value, e.i., unity. Reference 13 demonstrates a way to specify a new element spacing function. The adaptation procedure self-terminates as all elements of $\mathbf{I}_{s}$ become less than unity (i.e. no nodes are flagged for adaptation).

\section{Adaptation Mechanics}

The adaptation mechanics utilize three independent modules. The first module inserts new nodes into the existing mesh and locally reconnects tetrahedra and boundary faces to maintain a valid tessellation. The second module employs face and edge swapping to improve the mesh quality. The final module performs 
grid smoothing and boundary node projection operations.

\section{Node Insertion}

The node insertion method is currently one level of selective h-refinement. To start the refinement, all the edges surrounding nodes on the original mesh that have a scaled intensity $\mathbf{I}_{s}$ greater than unity are flagged for h-refinement. The set of flagged edges is examined tetrahedron by tetrahedron and additional edges are flagged in an attempt to maintain grid quality (i.e., low maximum dihedral angles, few high-degree nodes). The final set of flagged edges results in tetrahedra with one edge, three edges on one face, or all six edges flagged. A tetrahedron with all six edges flagged is illustrated in Fig. 1(b). The mesh is then h-refined by inserting new nodes on the midpoint of the flagged edges and reconnecting these nodes into new tetrahedra and boundary faces.

\section{Face and Edge Swapping}

The current post-adaptation grid-improvement scheme employs face and edge swapping. ${ }^{27}$ The swapping algorithm minimizes a shape (cost) function (e.g., aspect ratio or dihedral angle). This study sought to reduce only the cell aspect ratio $A R$

$$
A R=\frac{1}{3} \frac{\text { tetrahedral circumsphere radius }}{\text { tetrahedral in-sphere radius }}
$$

Reconnections of tetrahedra with undesirable shape measures are investigated and new local tetrahedra configurations with more desirable shape measures are selected. Edges on boundary faces can also be swapped. To simplify and speed up the edge swapping routine, the boundary face information is discarded and reconstructed at the end of the swapping process. Smart-Laplacian smoothing ${ }^{28}$ is used on the interior nodes. The actual locations of the boundary nodes is not modified in this module; that modification is performed by the grid smoothing and projection module.

\section{Grid Smoothing and Projection}

The inserted boundary nodes may not be located on the surface geometry of the model to be simulated since they were inserted at the midpoints of existing edges. A CAD model is employed to describe the actual model surface. To regain the surface fidelity of the mesh, the newly inserted boundary nodes are projected to the model surface with a CAD interface package CAPRI (Computational Analysis PRograming Interface).$^{29,30}$ The projection of these new nodes to their location on the CAD surface can result in inverted, invalid tetrahedral elements.

A grid node-smoothing algorithm is employed to facilitate boundary projection without generating invalid elements and to improve the overall quality (shape measure) of the mesh. This post-adaptation smoothing is similar to Freitag ${ }^{28}$ and was initially im- plemented by Brasher and Park. ${ }^{31}$ This initial implementation has been extended and coupled to CAPRI, which utilizes native CAD point projection routines. Nodes on the boundary are smoothed by moving the nodes in $\mathrm{CAD}(u, v)$ parametric space to improve the shape measure of adjacent tetrahedra.

As the nodes are projected, the neighboring tetrahedra are tested for validity. If invalid tetrahedra resulted from the projection, the projection distance of the boundary nodes is reduced until the neighboring tetrahedra are valid. Then the nodes in the neighborhood of the projected node are smoothed to improve a quality measure of the adjacent tetrahedra. The boundary points are then moved into the fully projected position in a number of iterative cycles.

It is anticipated that grid smoothing in the neighborhood of projected nodes may not adequately regain surface fidelity of anisotropic meshes. A gridmovement scheme may be required as in Ref. 16. Another possibility is a $3 \mathrm{D}$ version of mesh restructuring as in Ref. 32.

\section{Adaptation Module Interaction}

The current selective h-refinement technique often creates high-degree nodes on the border of the adapted regions. The smoothing algorithm is currently unable to improve elements that are adjacent to high-degree nodes. The edge and face swapping techniques effectively improve shape measures and reduce the number of high-degree nodes, facilitating projection and node smoothing.

These three adaptation modules where developed independently to facilitate a quick initial implementation and to investigate the strengths and weaknesses of each technique. Merging the abilities of these three separate modules will allow for more flexible modifications of grids (e.g., point insertion, point removal, and anisotropic elements). ${ }^{4,6}$

\section{Results}

Adaptation results are shown for a wing, wing-body, and high lift configurations. The wing is simulated with incompressible and transonic flow conditions. The wing-body and high lift configurations are simulated with subsonic flow.

\section{Initial Mesh Generator}

The initial meshes for these error prediction and adaptation studies are generated with the FELISA mesher $^{4}$ connected to CAD geometry by CAPRI through the GridEx ${ }^{33}$ framework. FELISA is a Delaunay mesh generator with an advancing-front method for inserting nodes. The GridEx framework is currently being developed at NASA Langley Research Center to link various grid generation and adaptation strategies to geometry through CAPRI. This framework is also utilized in a batch mode to perform uniform grid refinement studies. 


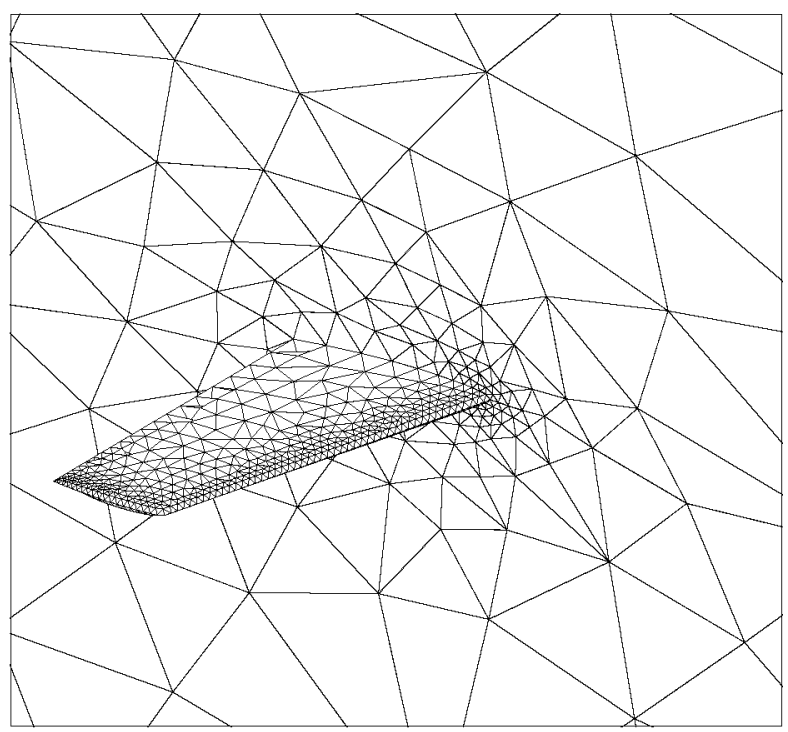

a) Initial ONERA M6 mesh.

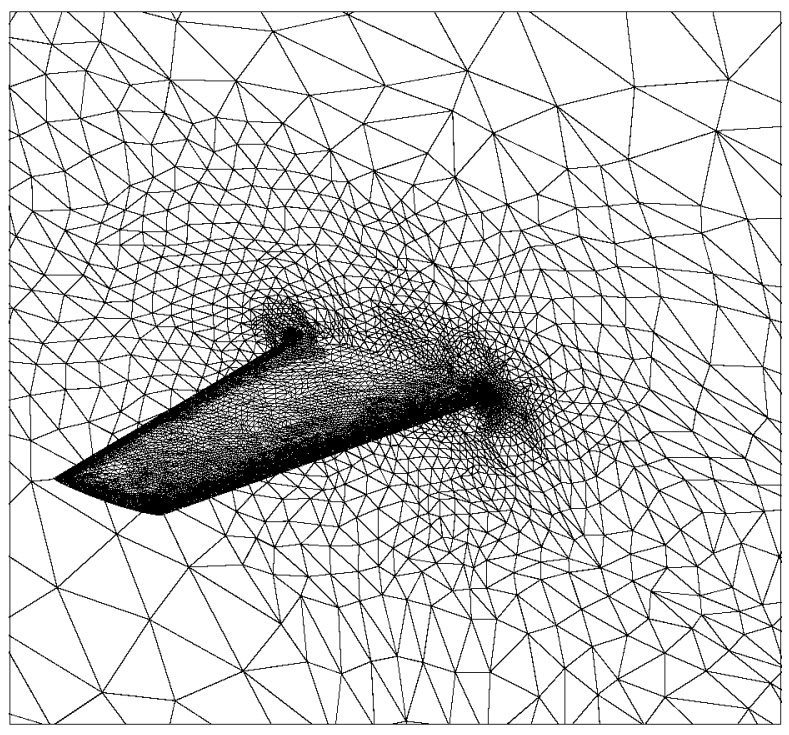

b) ONERA M6 mesh adapted to incompressible drag.

Fig. 3 Initial and adapted ONERA M6 meshes.

Drag Adaptation - Incompressible ONERA M6

The initial mesh for an ONERA (Office National d'Etudes et de Recherches Aérospatiales) M6 wing with 5227 nodes is shown in Fig. 3(a). The mesh has extremely coarse spacing, especially at the trailing edge and is intended to resolve the surface curvature of the leading edge and the wingtip and not any particular flow features. The spacing function for this mesh is specified manually and is intended to be representative of an automated curvature or maximum chord-height specification. The CAD geometry is represented with the CAPRI native kernel FELISA with a part generated from a surface IGES definition.

The initial ONERA M6 mesh was used in the grid adaptation process with incompressible flow at an an-

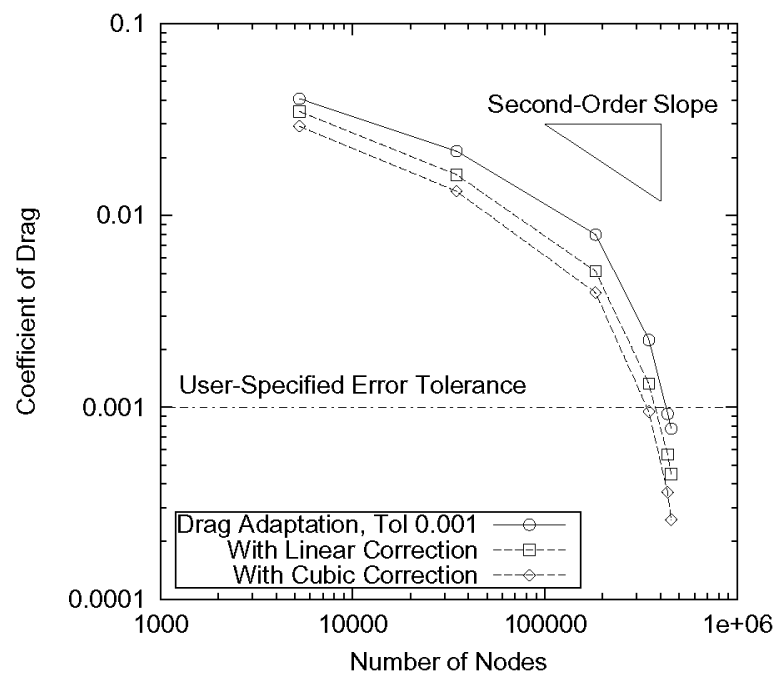

Fig. 4 Coefficient of drag for the ONERA M6 adapted to incompressible drag.

gle of attack of 0 deg. Directly computed drag and estimates of drag for an ONERA M6 wing as a function of number of nodes is shown in the Fig. $4 \log -\log$ plot. The adaptation and error correction results are shown for a drag error tolerance of 0.001 . The directly computed drag on the adapted meshes is represented by the solid lines with circular symbols. The errorcorrected drag calculated with the linear interpolated solution $f_{e s t}^{L}$ is represented by a dashed line and square symbols. The estimated functional calculated with the higher order interpolated solution $f_{\text {est }}^{H}$ is represented by a dashed line and diamond symbols. The correct drag is zero because of non-lifting, subcritical, inviscid flow. Therefore, the $y$-axis denoted "Coefficient of Drag" is also the error in drag.

The triangle labeled "Second-Order Slope" in Fig. 4 illustrates second-order spatial convergence. The adaptive-grid method results in drag calculations that converge at a much higher rate than the asymptotic convergence rate of uniform refinement (second-order). The adaptive procedure correctly selfterminated when the drag error of the adapted flow solution reached the user specified error tolerance (dotdash line).

The final grid (454 thousand nodes) after five cycles of grid adaptation to incompressible drag is shown in Fig. 3(b). The adaptation process clustered grid points at the leading and trailing edges of the wing. Points are also clustered in the neighborhood of the stagnation stream line.

The tetrahedra shape measure $A R$ (eq. 23) is minimized by the mesh improvement algorithm. The boundary node smoothing algorithm is intended to optimize the shape measures of the tetrahedral elements. Therefore, the shape measures of the boundary faces depicted in this surface plot may not be optimal. 


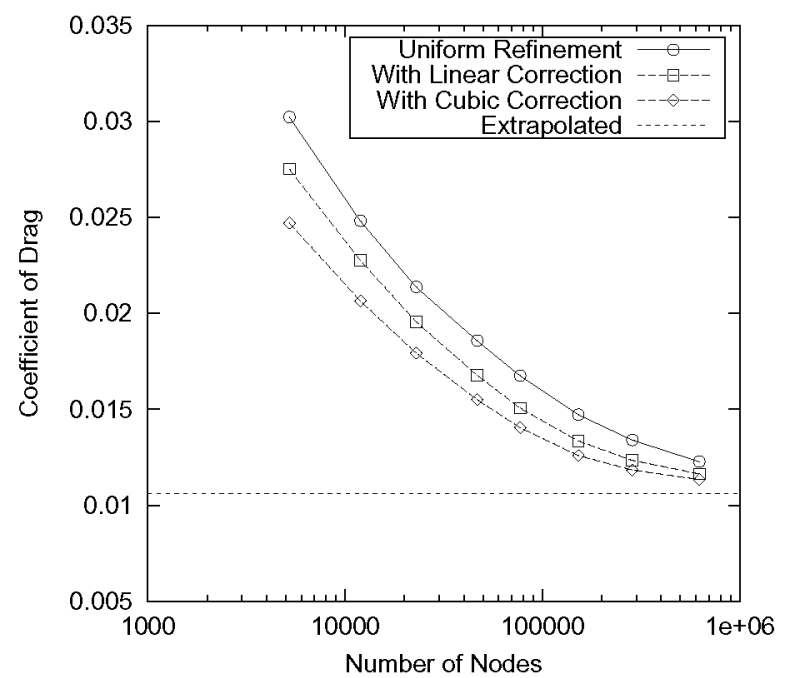

a) Uniformly refined drag convergence.

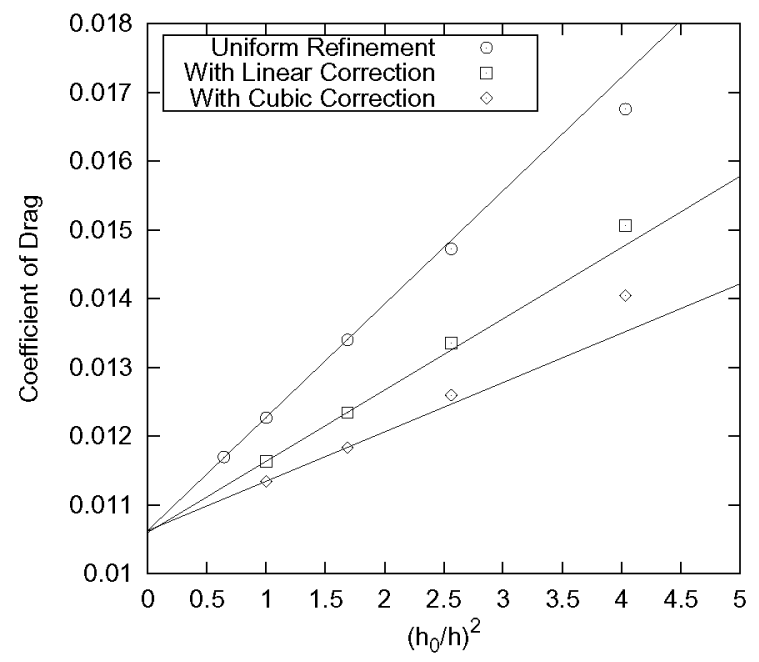

b) Richardson extrapolation.

Fig. 5 Coefficient of drag for the uniformly refined ONERA M6 at 0.84 Mach.

\section{Drag Adaptation - Transonic ONERA M6}

A uniform refinement of the ONERA M6 wing mesh is computed at 0.84 Mach and an angle of attack of 3 deg. The drag directly computed by the flow solver is shown with linear and higher order interpolated error corrections as a function of the number of nodes in Fig. 5(a). The extrapolated (grid-converged) drag value for Fig. 5(a) was estimated with Richardson extrapolation from Fig. 5(b). These meshes have the same spacing function as Fig. 3(a) globally modified with a scalar to uniformly reduce the element spacing. These grids were generated with the batch version of GridEx using the FELISA mesher and CAPRI for CAD geometry access.

Figure 5(b) shows drag and estimates of drag as a function of element size for the uniform grid re-

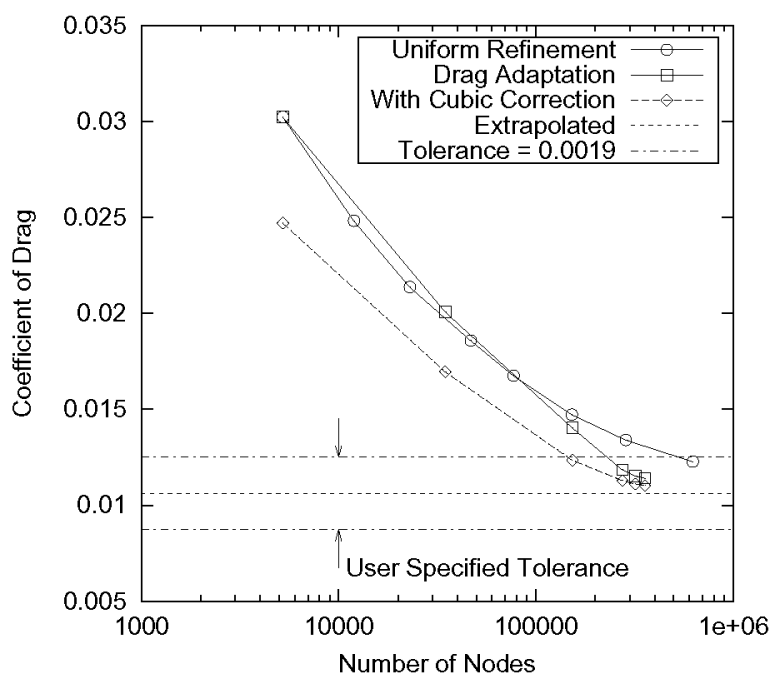

a) One level of h-refinement.

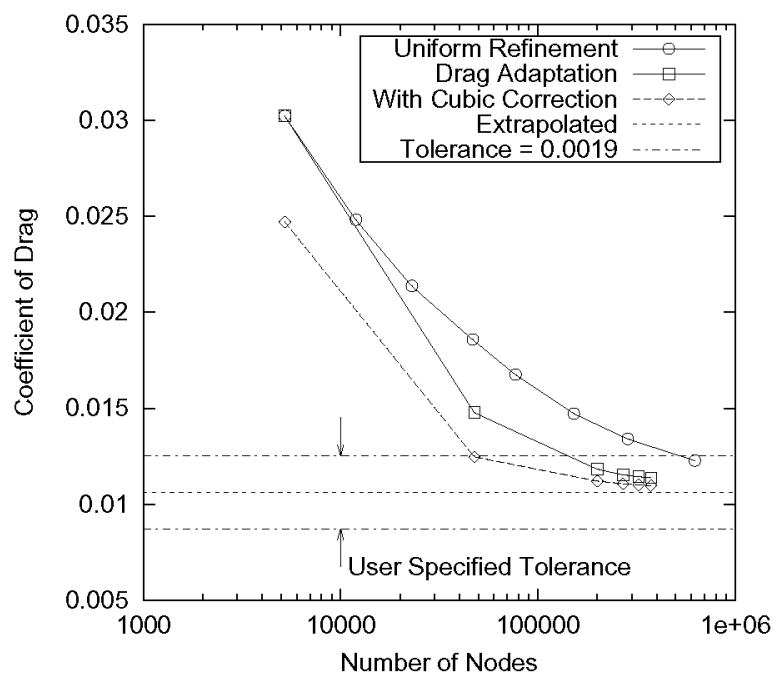

b) Two levels of h-refinement.

Fig. 6 Coefficient of drag for the drag adapted ONERA M6 at 0.84 Mach.

finement of the ONERA M6 wing. A representative element length $h$ was estimated by computing the cube root of the number of nodes. This length was normalized by the estimated length of the 624 thousand node mesh $h_{0}$. The symbols are drag computed by the flow solver and error corrected values. A linear fit of the data at $\left(h_{0} / h\right)^{2}=1.0$ and $\left(h_{0} / h\right)^{2}=1.7$ is used to estimate the grid-converged answer for all three schemes. All three schemes indicate a similar grid-resolved value. An additional flow solution (1.2 million nodes, $\left.\left(h_{0} / h\right)^{2}=0.6\right)$ is shown to verify the accuracy of the linear fit; it was not used to construct the linear fit of the computed drag. An improved interpolation scheme for the error correction would yield a superconvergent functional estimate as in Ref. 11.

The initial coarse mesh shown in Fig. $3(a)$ is em- 


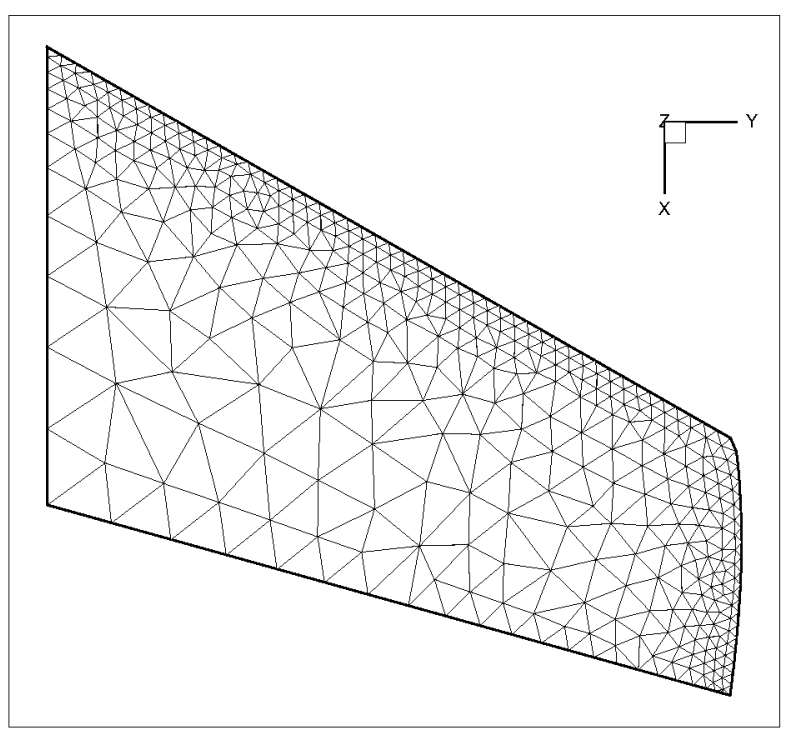

a) Upper wing surface mesh.

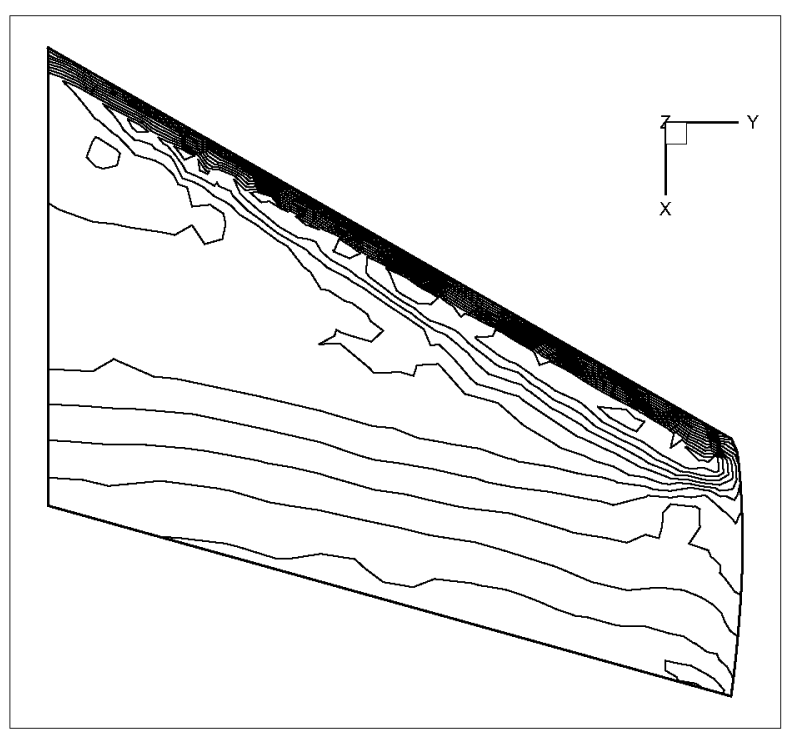

b) Upper wing surface Mach contours.

Fig. 7 Initial ONERA M6 upper wing surface.

ployed in the error prediction and grid adaptation procedure with two different adaptation methods. The coefficient of drag is plotted as a function of mesh size in Fig. 6. The user specified error tolerance in drag is 0.0019 . The uniformly refined flow solution from Fig. 5(a) is shown with the adapted grid flow solution and higher order error prediction of the adapted grid in Fig. 6. Figure 6(a) demonstrates a single level of h-refinement for all nodes with a scaled adaptation intensity $\mathbf{I}_{s}$ greater than one. Figure 6(b) shows hrefinement for $\mathbf{I}_{s}$ greater than one and a recursive call to the adaptation mechanics for $\mathbf{I}_{s}$ greater than 75 , yielding two levels of h-refinement at each adaptation cycle. The initial convergence of the function is better in Fig. 6(b) than Fig. 6(a). This improvement in func-

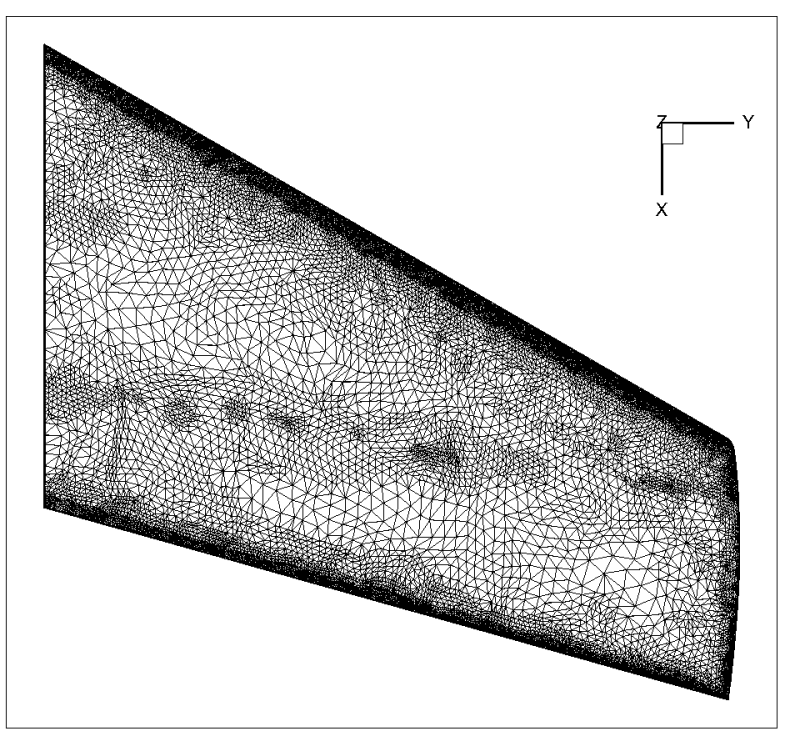

a) Upper wing surface mesh.

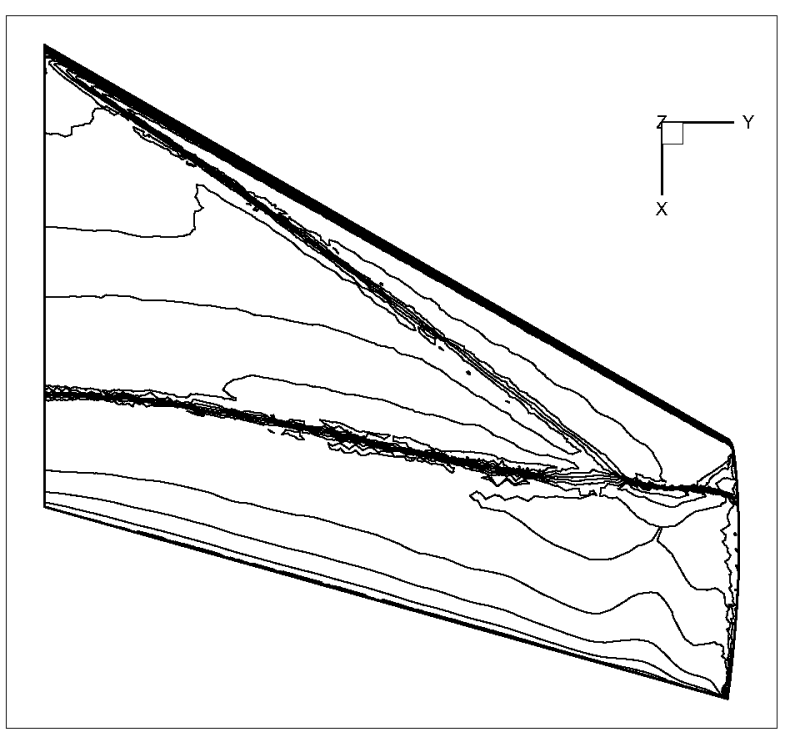

b) Upper wing surface Mach contours.

Fig. 8 Final ONERA M6 upper wing surface, adapted with one level of h-refinement.

tion convergence is illustrating the limitations of using a single level of selective h-refinement as the adaptive node-insertion procedure. The use of two levels of h-refinement better approximates a continuous variation in element size. The two adaptation methods converged to similar meshes and drag values.

The upper wing surface grid and Mach contours of the initial flow field computed on the mesh from Fig. 3(a) is shown in Fig. 7. The shocks in this initial grid are poorly resolved. The mesh (357 thousand nodes) and Mach contours of the ONERA M6 adapted to drag with one level of selective h-refinement is shown in Fig. 8. The mesh (374 thousand nodes) and Mach contours of the ONERA M6 adapted to 


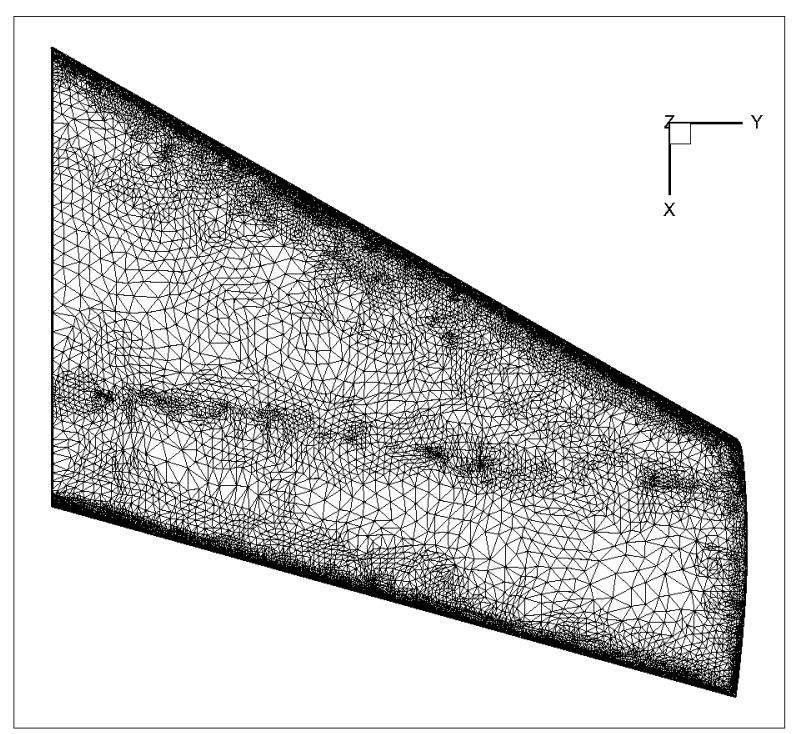

a) Upper wing surface mesh.

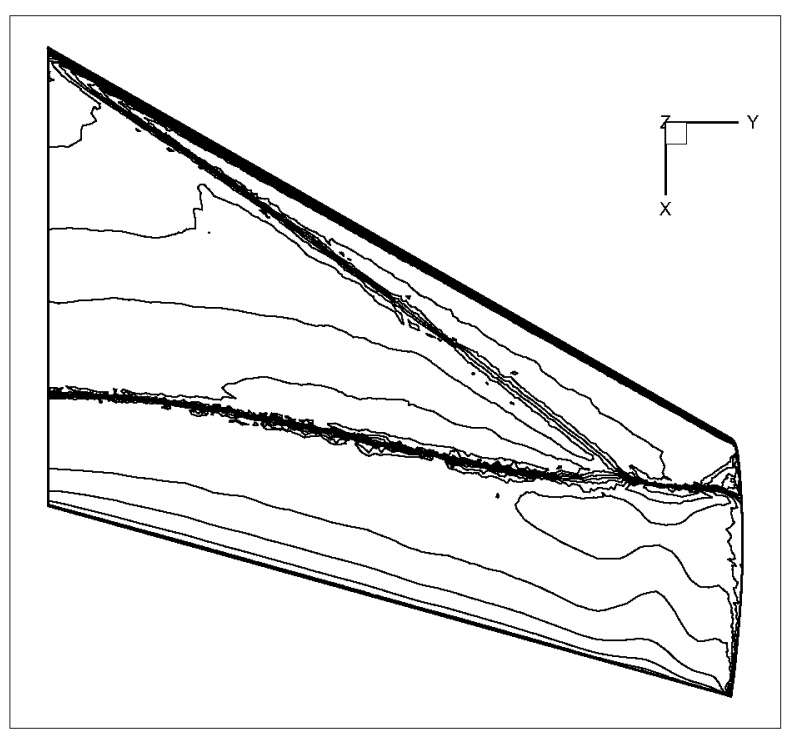

b) Upper wing surface Mach contours.

Fig. 9 Final ONERA M6 upper wing surface, adapted with two levels of h-refinement.

drag with two levels of selective h-refinement is shown in Fig. 8. The final meshes and solutions are similar for both of the adaptation methods. The adaptive procedure strongly clustered nodes at the leading and trailing edges of the wing and lightly clustered nodes at the shock location on the upper surface. Featurebased adaptations of this configuration in Ref. 7 and 8 focused on the leading edge and shock locations, but not the trailing edge.

\section{Lift Adaptation - EET}

The EET (Energy Efficient Transport) ${ }^{34}$ initial coarse mesh is shown in Fig. 10. The initial grid spacing distribution is specified manually to resolve the

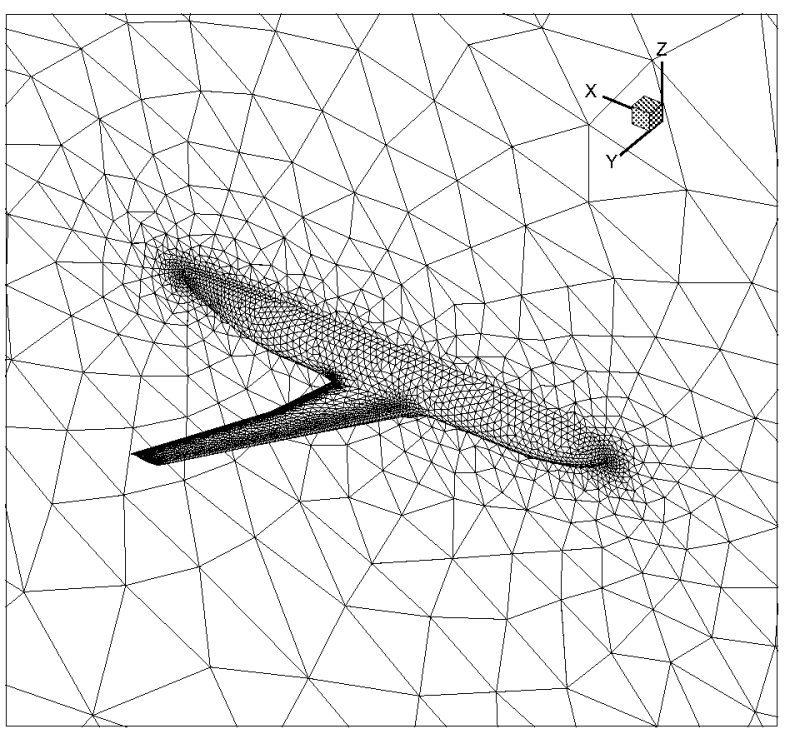

Fig. 10 Initial EET grid.

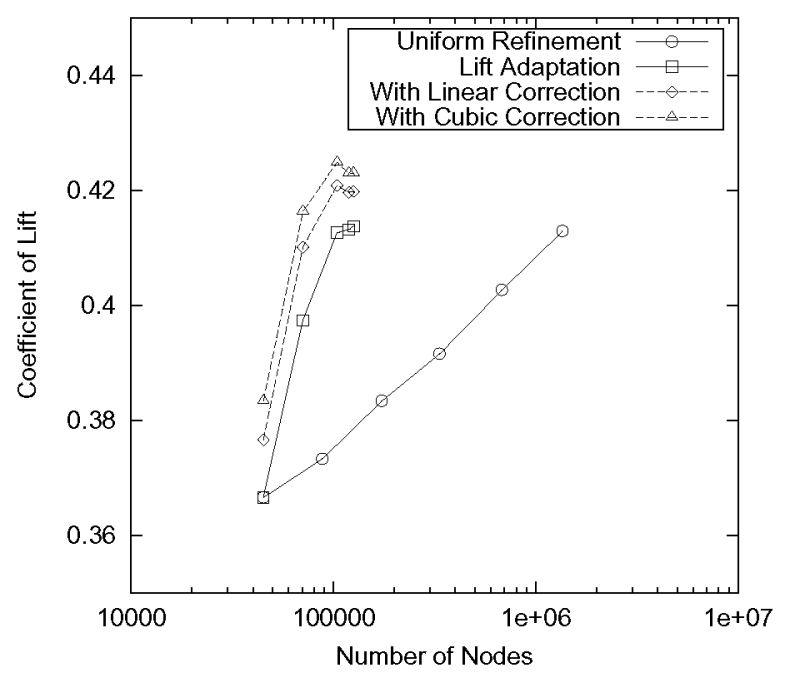

Fig. 11 Coefficient of lift for the lift-adapted EET at 0.40 Mach.

surface details of the fuselage, wing leading edge, blunt trailing edge, and the wingtip. The geometry is represented with a Parasolid CAD kernel accessed though the CAPRI application program interface (API).

The initial coarse mesh shown in Fig. 10 is employed in the lift error prediction and grid adaptation procedure at 0.40 Mach an angle of attack of 2 deg. The lift coefficient is plotted as a function of mesh size in Fig. 11. The adaptive procedure has a user specified error tolerance of 0.1 for the lift coefficient error. The uniformly refined lift calculation is shown with the adapted grid lift calculation and error predictions on the adapted grid in Fig. 11. The Richardson extrapolation value is not shown because a reasonable linear fit of the last three points was not possible. The lift coefficient is calculated on an adapted mesh one-tenth 


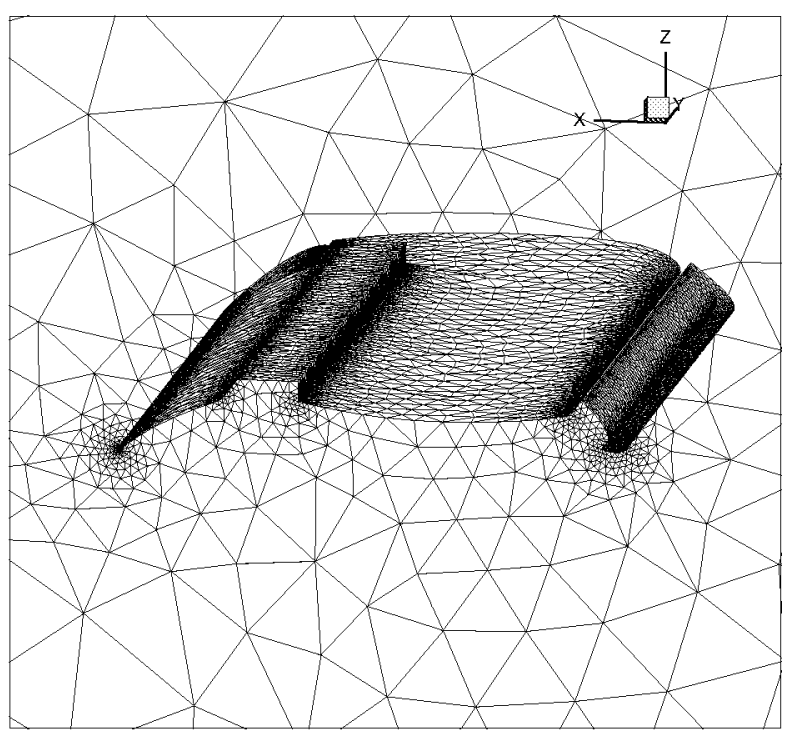

Fig. 12 Initial 30P-30N grid.

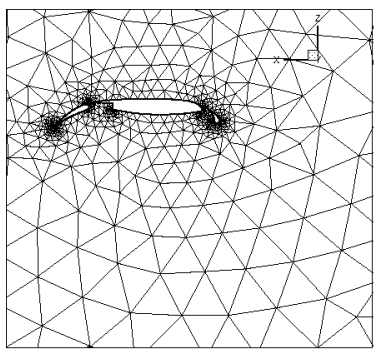

a) Initial $30 P-30 N$ grid.

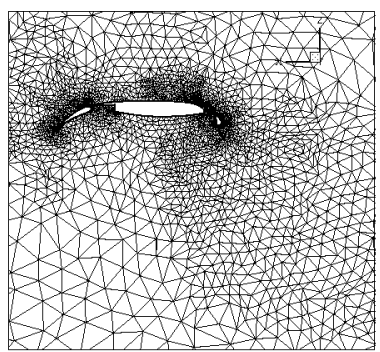

b) Lift-adapted 30P-30N grid.
Fig. 13 Original and adapted 30P-30N symmetry plane grids.

the size of the uniformly refined grid. The adapted lift error is well within the user specified tolerance.

\section{Lift Adaptation - 30P-30N Airfoil}

The McDonnell Douglas 30P-30N airfoil initial coarse mesh is shown in Fig. 12. The 30P-30N airfoil is extruded between two symmetry planes. The nearplane has been removed to improve visualization. The geometry is represented with a Parasolid CAD kernel accessed though the CAPRI API. This configuration is the subject of a recent $3 \mathrm{D}$ CFD study. ${ }^{35}$

The geometry and initial coarse mesh (113 thousand nodes) shown in Fig. 12 is employed in the error prediction and grid adaptation procedure at $0.20 \mathrm{Mach}$ and an angle of attack of $16.3 \mathrm{deg}$. The lift adaptive procedure has a user specified error in lift of 0.25 . The uniformly refined grid flow solution, adapted grid flow solution, linear error prediction, and higher order error prediction are shown in Fig. 14. The extrapolated coefficient of lift value was computed with a Richardson extrapolation of the finest two uniformly refined solutions. The original symmetry plane grid and the symmetry plane grid ( 832 thousand nodes) after two

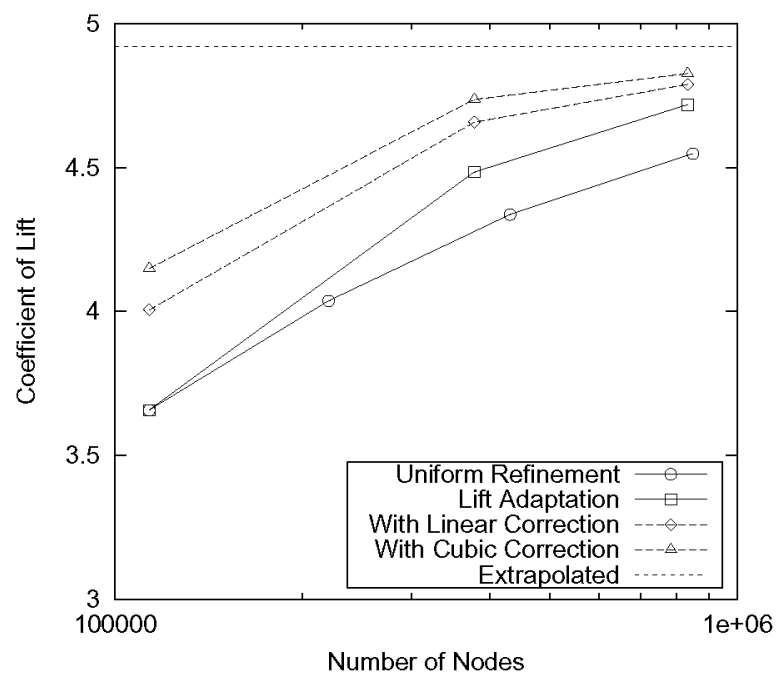

Fig. 14 Coefficient of lift for the lift-adapted 30P$30 \mathrm{~N}$ at $0.20 \mathrm{Mach}$.

cycles of adaptation is shown in Fig. 13.

Table 1 shows the aspect ratio $A R$ (eq. 23) for the initial and the adapted grids. The $A R$ is the cost

Table 1 Shape measure for the 30P-30N adaptation

\begin{tabular}{|c|c|c|}
\hline Cycle & Aspect ratio $A R$ & Face angle \\
\hline & $\max$ & $\max$ \\
\hline 1 & 8.1 & 154.3 \\
\hline 2 & 5.0 & 158.1 \\
\hline 3 & 7.5 & 167.8 \\
\hline
\end{tabular}

function for the grid-improvement optimizer. The face (dihedral) angle is not directly controlled but could be added as a constraint.

\section{Conclusion}

The initial implementation of an adjoint-based error correction and adaptation method has been demonstrated in three dimensions. With a given flow and adjoint solution, the error correction for a functional and adaptation intensity term have been described. The adaptation intensity was formulated to reduce the uncertainty in the error correction of a global functional and not a local error estimate as in a featurebased adaptation scheme. The adaptive procedure automatically terminates the simulation when an user specified tolerance is satisfied. The error remaining in the simulation at termination was always within the user specified tolerance, although sometimes the simulation was overly accurate.

A wing configuration was adapted to reduce drag error in incompressible and transonic flow. The drag computed by this adaptation and error correction method was shown to be as accurate as direct flow calculations using larger uniformly refined grids. The initial convergence of adaptation procedure improved 
with two levels of h-refinement at each adaptation cycle. Lift adaptations of Parasolid CAD models of wing-body and high lift configurations demonstrate the utility of this adaptive methodology on complex geometries. The lift of the wing-body configuration was computed on an adapted grid that is one-tenth the size of an uniformly refined grid.

\section{Acknowledgments}

The author wishes to thank Dr. Eric Nielsen for his help in employing the FUN3D flow and adjoint solvers, David Venditti and Dr. David Darmofal for their suggestions and guidance in error prediction and adaptation, Michael Brasher for his work researching and implementing mesh smoothing, William Jones for help with the GridEx framework, Dr. James Thomas for this support of this work, and the Fast Adaptive AeroSpace Tools (FAAST) development team.

\section{References}

${ }^{1}$ Peraire, J., Vahdati, M., Morgan, K., and C., Z. O., "Adaptive Remeshing for Compressible Flow Computations," Journal of Computational Physics, Vol. 72, 1987, pp. 449-266.

${ }^{2}$ Baker, T. J., "Mesh Adaptation Strategies for Problems in Fluid Dynamics," Finite Elements in Analysis and Design, Vol. 25, 1997, pp. 243-273.

${ }^{3}$ Mavriplis, D. J., "Adaptive Meshing Techniques for Viscous Flow Calculations on Mixed Element Unstructured Meshes," AIAA Paper 97-0857, 1997.

${ }^{4}$ Peraire, J. and Morgan, K., "Unstructured Mesh Generation Including Directional Refinement for Aerodynamic Flow Simulation," Finite Elements in Analysis and Design, Vol. 25, 1997, pp. 343-356.

${ }^{5}$ Braack, M. and Rannacher, R., "Adaptive Finite Element Methods for Low-Mach Flows with Chemical Reactions," Vol. 3 of 30th Computational Fluid Dynamics, von Karman Institute, 1999, pp. 1-93.

${ }^{6}$ Löhner, R., "Generation of Unstructured Grids Suitable for RANS Calculations," AIAA Paper 99-0662, 1999.

${ }^{7}$ Pirzadeh, S. Z., "A Solution-Adaptive Unstructured Grid Method by Grid Subdivision and Local Remeshing," AIAA Journal of Aircraft, Vol. 37, No. 5, September-October 2000, pp. 818-824, See also AIAA Paper 99-3255.

${ }^{8}$ Park, M. T. and Kwon, O. J., "Unsteady Flow Computations Using a 3-D Parallel Unstructured Dynamic Mesh Adaptation Algorithm," AIAA Paper 2001-0865, 2001.

${ }^{9}$ Monk, P. and Suli, E., "The Adaptive Computation of Far-Field Patterns by A Posteriori Error Estimation of Linear Functionals," SIAM Journal on Numerical Analysis, Vol. 8, 1998, pp. 251-274.

${ }^{10}$ Paraschivoiu, M., Peraire, J., and Patera, A., "A Posteriori Finite Element Bounds for Linear-functional Outputs of Elliptic Partial Differential Equations," Computer Methods in Applied Mechanics and Engineering, Vol. 150, 1997, pp. 289-312.

${ }^{11}$ Pierce, N. A. and Giles, M. B., "Adjoint Recovery of Superconvergent Functionals from PDE Approximations," SIAM Review, Vol. 42, No. 2, 2000, pp. 247-264.

${ }^{12}$ Venditti, D. A. and Darmofal, D. L., "Adjoint Error Estimation and Grid Adaptation for Functional Outputs: Application to Quasi-One-Dimensional Flow," Journal Computational Physics, Vol. 164, 2000, pp. 204-227, See also AIAA Paper 99 3292.

${ }^{13}$ Venditti, D. A. and Darmofal, D. L., "Grid Adaptation for Functional Outputs: Application to Two-Dimensional Inviscid Flows," Journal Computational Physics, Vol. 176, 2002, pp. 4069, See also AIAA Paper 2000-2244.
${ }^{14}$ Müller, J. D. and Giles, M. B., "Solution Adaptive Mesh Refinement Using Adjoint Error Analysis," AIAA Paper 20012550,2001

${ }^{15}$ Nielsen, E. J. and Anderson, W. K., "Recent Improvements in Aerodynamic Design Optimization on Unstructured Meshes," AIAA Paper 2001-0596, 2001.

${ }^{16}$ Nielsen, E. J. and Anderson, W. K., "Aerodynamic Design Optimization On Unstructured Meshes Using the Navier-Stokes Equations," AIAA Paper 98-4809, 1998.

${ }^{17}$ Anderson, W. K., Rausch, R. D., and Bonhaus, D. L., "An Implicit Upwind Algorithm for Computing Turbulent Flows on Unstructured Grids," Computers and Fluids, Vol. 23, No. 1, 1994, pp. 1-22.

${ }^{18}$ Anderson, W. K. and Bonhaus, D. L., "Implicit/Multigrid Algorithm for Incompressible Turbulent Flows on Unstructured Grids," AIAA Paper 95-1740, 1995.

${ }^{19}$ Nielsen, E. J., Aerodynamic Design Sensitivities on an Unstructured Mesh Using the Navier-Stokes Equations and a Discrete Adjoint Formulation, Ph.D. thesis, Virginia Polytechnic Institute and State University, 1998.

${ }^{20}$ Spalart, P. R. and Allmaras, S. R., "One-Equation Turbulence Model for Aerodynamic Flows," AIAA Paper 92-0429, 1992.

${ }^{21}$ Fast Adaptive AeroSpace Tools (FAAST) development team, "Opportunities for Breakthoughs in Large-Scale Computaional Simulation and Design," To be published as a NASA Technical Memorandum, NASA Langley Research Center, 2002.

${ }^{22}$ Taylor, A. C., Green, L. L., Newman, P. A., and Putko, M. M., "Some Advanced Concepts in Discrete Aerodynamic Sensitivity Analysis," AIAA Paper 2001-2529, 2001.

${ }^{23}$ Saad, Y. and Schultz, M. H., "GMRES: A Generalized Minimum Residual Algorithm for Solving Nonsymmetric Linear Systems," SIAM Journal of Scientific and Statistical Computing, Vol. 7, 1986, pp. 856-869.

${ }^{24}$ Giles, M., "On the use of Runge-Kutta time-marching and multigrid for the solution of the steady adjoint equations," Technical Report NA00/10, 2000.

${ }^{25}$ Giles, M., "Adjoint Code Developments Using the Exact Discrete Approach," AIAA Paper 2001-2596, 2001.

${ }^{26} \mathrm{Lu}$, J. and Darmofal, D., Private Communication, Massachusetts Institute of Technology.

${ }^{27}$ Freitag, L. A. and Ollivier-Gooch, C., "Tetrahedral Mesh Improvement Using Swapping and Smoothing," International Journal for Numerical Methods in Engineering, Vol. 40, 1997, pp. 3979-4002.

${ }^{28}$ Freitag, L. A., "On Combining Laplacian and Optimization-Based Smoothing Techniques," ASME, Vol. 220, July 1997 , pp. 37-43.

${ }^{29}$ Haimes, R., "CAPRI: Computational Analysis PRogramming Interface," Proceedings of the 6th International Conference on Numerical Grid Generation in Computational Field Simulations, July 1998.

${ }^{30}$ Haimes, R., "Automatic Generation of CFD-Ready Surface Triangulations from CAD Geometry," AIAA Paper 99-0776, 1999.

${ }^{31}$ Brasher, M. L. and Park, M. A., "Isotropic Smoothing of 3D Unstructured Tetrahedral Meshes," LARSS Final Report, NASA Langley Research Center, 2001.

${ }^{32}$ Mavriplis, D., "Turbulent Flow Calculations Using Unstructured and Adaptive Meshes," ICASE Report No. 90-61, NASA CR-182102, 1990.

${ }^{33}$ Jones, W. T., "An Open Framework for Unstructured Grid Generation," AIAA Paper 2002-3192, 2002.

${ }^{34} \mathrm{Jacobs}$, P. F. and Gloss, B. B., "Longitudinal Aerodynamic Characteristics of a Subsonic, Energy-Efficient Transport Configuration in the National Transonic Facility," TP 2922, NASA Langley Research Center, Aug. 1989.

${ }^{35}$ Rumsey, C. L., Lee-Rausch, E. M., and Watson, R. D., "Three-Dimensional Effects on Multi-Element High Lift Computations," AIAA Paper 2002-0845, 2002. 